\title{
Pacific
}

Journal of

Mathematics

\section{HYPERGEOMETRIC EVALUATION IDENTITIES AND SUPERCONGRUENCES}

\author{
LING LONG
}




\title{
HYPERGEOMETRIC EVALUATION IDENTITIES AND SUPERCONGRUENCES
}

\author{
LING LONG
}

\begin{abstract}
We apply some hypergeometric evaluation identities, including a strange valuation of Gosper, to prove several supercongruences related to special valuations of truncated hypergeometric series. In particular, we prove a conjecture of van Hamme.
\end{abstract}

\section{Introduction}

In this article, we use $p$ to denote an odd prime. Zudilin [2009] proved several Ramanujan-type supercongruences using the Wilf-Zeilberger (WZ) method. One of them, conjectured by van Hamme, says that

$$
\sum_{k=0}^{(p-1) / 2}(4 k+1)\left(\frac{\left(\frac{1}{2}\right) k}{k !}\right)^{3}(-1)^{k} \equiv(-1)^{(p-1) / 2} p \bmod p^{3},
$$

where $(a)_{k}=a(a+1) \cdots(a+k-1)$ is the rising factorial for $a \in \mathbb{C}$ and $k \in \mathbb{N}$.

The first proof of (1) was given by Mortenson [2008]. It is said to be of Ramanujan-type because it is a $p$-adic version of Ramanujan's formula

$$
\sum_{k=0}^{\infty}(4 k+1)\left(\frac{\left(\frac{1}{2}\right)_{k}}{k !}\right)^{3}(-1)^{k}=\frac{2}{\pi} .
$$

See [Zudilin 2009] for more Ramanujan-type supercongruences.

In this short note, we will present a new proof of (1), which summarizes our strategy in proving similar types of supercongruences.

McCarthy and Osburn [2008] proved van Hamme's conjecture [1997] that

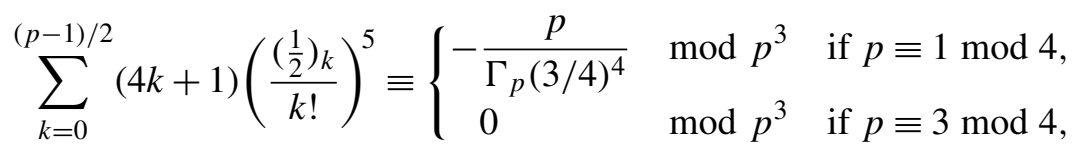

where $\Gamma_{p}(\cdot)$ denotes the $p$-adic Gamma function.

Supported by NSA grant H98230-08-1-0076.

MSC2000: 33C20.

Keywords: Ramanujan supercongruences, hypergeometric identities. 
Similarly, van Hamme has conjectured that for any prime $p>3$,

$$
\sum_{k=0}^{(p-1) / 2}(6 k+1)\left(\frac{\left(\frac{1}{2}\right)_{k}}{k !}\right)^{3} 4^{-k} \equiv(-1)^{(p-1) / 2} p \bmod p^{4} .
$$

This formula is supported by numerical evidence, but as van Hamme said, "we have no real explanation for our observations". In our exploration, it will become clear that such supercongruences are a result of extra symmetries, which we are able to interpret using hypergeometric evaluation identities. Of course, they can also be seen from other perspectives, such as the WZ method.

Meanwhile, it is known that some of the truncated hypergeometric series are related to the number of rational points on certain algebraic varieties over finite fields and further to coefficients of modular forms. For instance, based on the result of Ahlgren and Ono [2000], Kilbourn [2006] proved that

$$
\sum_{k=0}^{(p-1) / 2}\left(\frac{\left(\frac{1}{2}\right)_{k}}{k !}\right)^{4} \equiv a_{p} \bmod p^{3},
$$

where $a_{p}$ is the $p$-th coefficient of a weight 4 modular form

$$
\eta(2 z)^{4} \eta(4 z)^{4}:=q \prod_{n \geq 1}\left(1-q^{2 n}\right)^{4}\left(1-q^{4 n}\right)^{4}, \quad \text { where } q=e^{2 \pi i z} .
$$

This is one instance of the supercongruences conjectured by Rodriguez-Villegas [2003], which relate special truncated hypergeometric series values and coefficients of Heck eigenforms. McCarthy [2009] proved another supercongruence of this type and his approach provides a general combinatorial framework for all these congruences.

We will establish a few supercongruences mainly via hypergeometric evaluation identities and combinatorics. Since there exist many amazing hypergeometric evaluation identities in the literature, we expect that our approach can be used to prove other interesting congruences.

Here is a summary of our results.

Theorem 1.1. Let $p>3$ be a prime and $r$ be a positive integer. Then

$$
\sum_{k=0}^{\left(p^{r}-1\right) / 2}(4 k+1)\left(\frac{\left(\frac{1}{2}\right)_{k}}{k !}\right)^{4} \equiv p^{r} \bmod p^{3+r} .
$$

Theorem 1.2. Let $p>3$ be a prime. Then

$$
\sum_{k=0}^{(p-1) / 2}(4 k+1)\left(\frac{\left(\frac{1}{2}\right)_{k}}{k !}\right)^{6} \equiv p \cdot a_{p} \quad \bmod p^{4} .
$$


Conjecture 1.3. Let $p>3$ be a prime and $r$ be a positive integer. Then

$$
\sum_{k=0}^{\left(p^{r}-1\right) / 2}(4 k+1)\left(\frac{\left(\frac{1}{2}\right)_{k}}{k !}\right)^{6} \equiv p^{r} \cdot a_{p^{r}} \bmod p^{3+r},
$$

where $a_{p^{r}}$ is the $p^{r}$-th coefficient of (4).

Theorem 1.4. Van Hamme's conjecture (2) is true.

Theorem 1.5. Let $p>3$ be a prime. Then

$$
\sum_{k=0}^{(p-1) / 2}(6 k+1)\left(\frac{\left(\frac{1}{2}\right)_{k}}{k !}\right)^{3} \frac{(-1)^{k}}{8^{k}} \equiv(-1)^{\left(p^{2}-1\right) / 8+(p-1) / 2} p \quad \bmod p^{2} .
$$

\section{Preliminaries}

Hypergeometric series. For any positive integer $r$,

$$
{ }_{r+1} F_{r}\left[\begin{array}{c}
a_{1}, a_{2}, \ldots, a_{r+1} ; z \\
b_{1}, \ldots, b_{r}
\end{array}\right]=\sum_{k \geq 0} \frac{\left(a_{1}\right)_{k} \cdots\left(a_{r+1}\right)_{k}}{k !\left(b_{1}\right)_{k} \cdots\left(b_{r}\right)_{k}} z^{k},
$$

where $(a)_{k}$ is the rising factorial and $z \in \mathbb{C}$. A hypergeometric series terminates if it is well-defined and at least one of the $a_{i}$ is a negative integer. We will make use of this fact to produce various truncated hypergeometric series.

By the definition of the rising factorial,

$$
\frac{\left(\frac{1}{2}\right)_{k}}{k !}=2^{-2 k}\left(\begin{array}{c}
2 k \\
k
\end{array}\right) .
$$

Gamma function. Let $\Gamma(x)$ denote the usual Gamma function, which is defined for all $x \in \mathbb{C}$ except for the nonpositive integers. It satisfies some well known properties, such as $\Gamma(x+1)=x \Gamma(x)$. Thus, $(a)_{k}=\Gamma(a+k) / \Gamma(a)$ when $\Gamma(a) \neq 0$ and $\Gamma(a+k)$ are defined.

Another formula we need is Euler's reflection formula

$$
\Gamma(x) \Gamma(1-x)=\frac{\pi}{\sin (\pi x)} .
$$

Some combinatorics. We gather here some results in combinatorics to be used later. It is the author's pleasure to acknowledge that the approaches used in (7)(10) are due to Zudilin. Here is a key idea of Zudilin for rising factorials; see also [Chan et al. 2010, Lemma 1]:

$$
\begin{aligned}
\left(\frac{1}{2}+\varepsilon\right)_{k} & =\left(\frac{1}{2}+\varepsilon\right)\left(\frac{1}{2}+\varepsilon+1\right) \cdots\left(\frac{1}{2}+\varepsilon+k-1\right) \\
& =\left(\frac{1}{2}\right)_{k}\left(1+2 \varepsilon \sum_{j=1}^{k} \frac{1}{2 j-1}+4 \varepsilon^{2} \sum_{1 \leq i<j \leq k}^{k} \frac{1}{(2 i-1)(2 j-1)}+O\left(\varepsilon^{3}\right)\right) .
\end{aligned}
$$


Hence, $\left(\frac{1}{2}+\varepsilon\right)_{k}\left(\frac{1}{2}-\varepsilon\right)_{k}$ can be expanded as a power series of $\varepsilon^{2}$ as

$$
\left(\frac{1}{2}+\varepsilon\right)_{k}\left(\frac{1}{2}-\varepsilon\right)_{k}=\left(\frac{1}{2}\right)_{k}^{2}\left(1-4 \varepsilon^{2} \sum_{j=1}^{k} \frac{1}{(2 j-1)^{2}}+O\left(\varepsilon^{4}\right)\right) .
$$

Similarly,

$$
(1+\varepsilon)_{k}(1-\varepsilon)_{k}=(1)_{k}^{2}\left(1-\varepsilon^{2} \sum_{j=1}^{k} \frac{1}{j^{2}}+O\left(\varepsilon^{4}\right)\right) .
$$

Letting $\varepsilon=-p^{r} / 2$ and $\varepsilon=p^{r} / 2$ respectively in (7) and taking $k$ to be an integer between 1 and $\left(p^{r}-1\right) / 2$, we obtain

$$
(-1)^{k}\left(\begin{array}{c}
\left(p^{r}-1\right) / 2 \\
k
\end{array}\right) \equiv \frac{\left(\frac{1}{2}\right)_{k}}{k !} \bmod p \quad \text { and } \quad\left(\begin{array}{c}
\left(p^{r}-1\right) / 2+k \\
k
\end{array}\right) \equiv \frac{\left(\frac{1}{2}\right)_{k}}{k !} \bmod p .
$$

Similarly, letting $\varepsilon=p^{r} / 2$ in (8) and $k$ be an integer between 1 and $\left(p^{r}-1\right) / 2$, we have

$$
(-1)^{k}\left(\begin{array}{c}
\left(p^{r}-1\right) / 2 \\
k
\end{array}\right)\left(\begin{array}{c}
\left(p^{r}-1\right) / 2+k \\
k
\end{array}\right) \equiv\left(\frac{\left(\frac{1}{2}\right)_{k}}{k !}\right)^{2} \bmod p^{2} .
$$

Lemma 2.1. For any positive integer $n>1$,

$$
(2 n+1) \sum_{k=0}^{n} \frac{1}{2 k+1}\left(\begin{array}{l}
n \\
k
\end{array}\right)\left(\begin{array}{c}
n+k \\
k
\end{array}\right)(-1)^{k}=1 .
$$

Proof. We use the partial fraction decomposition

$$
\frac{(t-1)(t-2) \cdots(t-n)}{t(t+1) \cdots(t+n)}=\sum_{k=0}^{n}(-1)^{n-k}\left(\begin{array}{l}
n \\
k
\end{array}\right)\left(\begin{array}{c}
n+k \\
k
\end{array}\right) \frac{1}{t+k} .
$$

Letting $t=1 / 2$, this becomes

$$
(-1)^{n} \frac{2}{2 n+1}=2 \sum_{k=0}^{n}(-1)^{n-k}\left(\begin{array}{l}
n \\
k
\end{array}\right)\left(\begin{array}{c}
n+k \\
k
\end{array}\right) \frac{1}{1+2 k},
$$

which is equivalent to the claim of the lemma.

Lemma 2.2. Let $n$ be an odd positive integer. Then

$$
\frac{\left(\frac{3}{2}-\frac{1}{4} n\right)_{(n-1) / 2}\left(1-\frac{1}{2} n\right)_{(n-1) / 2}}{\left(2-\frac{1}{2} n\right)_{(n-1) / 2}\left(1-\frac{1}{4} n\right)_{(n-1) / 2}}=(-1)^{(n-1) / 2} n .
$$


Proof. Using $(a)_{k}=\Gamma(a+k) / \Gamma(a)$, we have

$$
\begin{aligned}
& \frac{\left(\frac{3}{2}-\frac{1}{4} n\right)_{(n-1) / 2}\left(1-\frac{1}{2} n\right)_{(n-1) / 2}}{\left(2-\frac{1}{2} n\right)_{(n-1) / 2}\left(1-\frac{1}{4} n\right)_{(n-1) / 2}} \\
& =\frac{\Gamma\left(\frac{3}{2}-\frac{1}{4} n+\frac{1}{2}(n-1)\right) \Gamma\left(\frac{1}{2}\right) \Gamma\left(2-\frac{1}{2} n\right) \Gamma\left(1-\frac{1}{4} n\right)}{\Gamma\left(\frac{3}{2}-\frac{1}{4} n\right) \Gamma\left(1-\frac{1}{2} n\right) \Gamma\left(\frac{3}{2}\right) \Gamma\left(1-\frac{1}{4} n+\frac{1}{2}(n-1)\right)} \\
& =\frac{\left(1-\frac{1}{2} n\right)}{\frac{1}{2}} \frac{\frac{1}{4} n \cdot \Gamma\left(\frac{1}{4} n\right) \Gamma\left(1-\frac{1}{4} n\right)}{\left(\frac{1}{2}-\frac{1}{4} n\right) \cdot \Gamma\left(\frac{1}{2}+\frac{n}{4}\right) \Gamma\left(\frac{1}{2}-\frac{1}{4} n\right)} \\
& =n \cdot \frac{\sin (\pi / 2-\pi n / 4)}{\sin (\pi n / 4)}=n \cdot \cot (\pi n / 4)=(-1)^{(n-1) / 2} n .
\end{aligned}
$$

Lemma 2.3. Let $n$ be an odd integer. Then

$$
\frac{\left(\frac{3}{2}-\frac{1}{4} n\right)_{(n-1) / 2}}{\left(2-\frac{1}{2} n\right)_{(n-1) / 2}} 2^{(n-1) / 2}=(-1)^{\left(n^{2}-1\right) / 8+(n-1) / 2} n .
$$

Proof. We have

$$
\frac{\left(\frac{3}{2}-\frac{1}{4} n\right)_{(n-1) / 2}}{\left(2-\frac{1}{2} n\right)_{(n-1) / 2}} 2^{(n-1) / 2}=\frac{\left(3-\frac{1}{2} n\right)\left(5-\frac{1}{2} n\right) \cdots \frac{n}{2}}{\left(2-\frac{1}{2} n\right)\left(3-\frac{1}{2} n\right) \cdots \frac{1}{2}}=\operatorname{sgn} \cdot n,
$$

where $\operatorname{sgn}=(-1)^{\#}$ and \# is the number of negative terms appearing in the fraction above. It is easy to see that

$$
\#=\left\lfloor\frac{1}{2}\left(\frac{1}{2} n+1\right)\right\rfloor+\left\lfloor\frac{1}{2} n\right\rfloor-2 \equiv \frac{1}{8}\left(n^{2}-1\right)+\frac{1}{2}(n-1) \quad \bmod 2 .
$$

Lemma 2.4 [Cai 2002]. For any prime $p>3$ and positive integer $r$,

$$
(-1)^{\left(p^{r}-1\right) / 2}\left(\begin{array}{c}
p^{r}-1 \\
\frac{1}{2}\left(p^{r}-1\right)
\end{array}\right) \equiv\left(\frac{\left(\frac{1}{2}\right)\left(p^{r}-1\right) / 2}{\left(\frac{1}{2}\left(p^{r}-1\right)\right) !}\right)^{2} \bmod p^{3} .
$$

Using (6), the congruence (11) is equivalent to

$$
\left(\begin{array}{c}
p^{r}-1 \\
\frac{1}{2}\left(p^{r}-1\right)
\end{array}\right) \equiv(-1)^{\left(p^{r}-1\right) / 2} 2^{2\left(p^{r}-1\right)} \bmod p^{3} .
$$

When $r=1$, this was proved in [Morley 1895].

A generalized harmonic sum. Let $H_{k}^{(2)}:=\sum_{j=1}^{k} \frac{1}{j^{2}}$.

Lemma 2.5 [Morley 1895]. Let $p>3$ be a prime. We have

$$
H_{(p-1) / 2}^{(2)} \equiv 0 \bmod p \quad \text { and } \quad \sum_{j=1}^{(p-1) / 2} \frac{1}{(2 j-1)^{2}} \equiv 0 \bmod p \text {. }
$$


Using arguments in [Morley 1895] or elementary congruence, it is easy to see the following lemma holds.

Lemma 2.6. Let $p>3$ be a prime. Then for every integer $k$ between 1 and $p-2$,

$$
H_{k}^{(2)}+H_{p-1-k}^{(2)} \equiv 0 \bmod p .
$$

Lemma 2.7. Let $p>3$ be a prime and $s$ be a positive integer. Then

$$
\sum_{k=0}^{(p-1) / 2}\left(\frac{\left(\frac{1}{2}\right)_{k}}{k !}\right)^{2 s} \cdot H_{2 k}^{(2)} \equiv 0 \bmod p .
$$

Proof. Using the fact that

$$
(-1)^{k}\left(\begin{array}{c}
\frac{1}{2}(p-1) \\
k
\end{array}\right) \equiv \frac{\left(\frac{1}{2}\right)_{k}}{k !} \bmod p,
$$

we have

$$
\begin{aligned}
& \sum_{k=0}^{(p-1) / 2}\left(\frac{\left(\frac{1}{2}\right)_{k}}{k !}\right)^{2 s} H_{2 k}^{(2)} \equiv \sum_{k=0}^{(p-1) / 2}\left(\begin{array}{c}
\frac{1}{2}(p-1) \\
k
\end{array}\right)^{2 s} H_{2 k}^{(2)} \bmod p \\
& \quad=\frac{1}{2}\left(\sum_{k=0}^{(p-1) / 2}\left(\begin{array}{c}
\frac{1}{2}(p-1) \\
k
\end{array}\right)^{2 s} H_{2 k}^{(2)}+\sum_{k=0}^{(p-1) / 2}\left(\begin{array}{c}
\frac{1}{2}(p-1) \\
\frac{1}{2}(p-1)-k
\end{array}\right)^{2 s} H_{p-1-2 k}^{(2)}\right) \\
& \quad=\frac{1}{2}\left(\sum_{k=0}^{(p-1) / 2}\left(\begin{array}{c}
\frac{1}{2}(p-1) \\
k
\end{array}\right){ }^{2 s}\left(H_{2 k}^{(2)}+H_{p-1-2 k}^{(2)}\right)\right) \\
& \equiv 0 \bmod p .
\end{aligned}
$$

2.1. An elementary p-adic analysis. Let $F\left(x_{1}, \ldots, x_{t} ; z\right)$ be a $(t+1)$-variable formal power series. For instance, it could be a scalar multiple of a terminating hypergeometric series as follows:

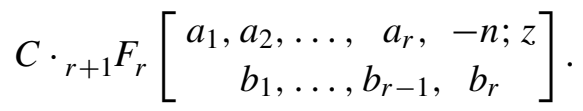

Assume that by specifying values $x_{i}=a_{i}$ for $i=1, \ldots, t$ and $z=z_{0}$, we have

$$
F\left(a_{1}, \ldots, a_{t} ; z_{0}\right) \in \mathbb{Z}_{p} .
$$

Now we fix $z_{0}$ and deform the parameters $a_{i}$ into polynomials $a_{i}(x) \in \mathbb{Z}_{p}[x]$ such that $a_{i}(0)=a_{i}$ for all $1 \leq i \leq t$, and assume that the resulting function $F\left(a_{1}(x), \ldots, a_{t}(x) ; z_{0}\right)$ is a formal power series in $x^{2}$ with coefficients in $\mathbb{Z}_{p}$, that is, $F\left(a_{1}(x), \ldots, a_{t}(x) ; z_{0}\right)=A_{0}+A_{2} x^{2}+A_{4} x^{4}+\cdots$ for $A_{i} \in \mathbb{Z}_{p}$, where $A_{0}=F\left(a_{1}, \ldots, a_{t} ; z_{0}\right)$. 
Lemma 2.8. Under the setting above, if $p^{s} \mid A_{2}$ for $s=1,2$, then

$$
F\left(a_{1}(p), \ldots, a_{t}(p) ; z_{0}\right) \equiv A_{0} \bmod p^{2+s} .
$$

\section{A new proof of (1)}

We briefly outline our method for proving the next few supercongruences; we are motivated by [McCarthy and Osburn 2008] and [Mortenson 2008]. To each congruence, we first identify a corresponding hypergeometric evaluation identity, which with specified parameters is congruent to a target truncated hypergeometric series evaluation up to some power of $p$. Usually the power of $p$ so obtained is weaker than the conjectural exponent. In our cases, we reduce the optimal congruences to some congruence combinatorial identities, which are established using additional hypergeometric evaluation identities or combinatorics.

Our strategy can be best implemented in the following new proof of (1). An identity of Whipple [1926, (5.1)] says

$$
{ }_{4} F_{3}\left[\begin{array}{ccc}
a, 1+a / 2, & c, & d ; \\
a / 2, & 1+a-c, 1+a-d
\end{array}\right]=\frac{\Gamma(1+a-c) \Gamma(1+a-d)}{\Gamma(1+a) \Gamma(1+a-c-d)} .
$$

Letting $a=\frac{1}{2}, c=\frac{1}{2}+\frac{1}{2} p$ and $d=\frac{1}{2}-\frac{1}{2} p$, we conclude immediately that

$$
\sum_{k=0}^{(p-1) / 2}(4 k+1)\left(\frac{\left(\frac{1}{2}\right)_{k}}{k !}\right)^{3}(-1)^{k} \equiv \frac{\Gamma\left(1-\frac{1}{2} p\right) \Gamma\left(1+\frac{1}{2} p\right)}{\Gamma\left(\frac{1}{2}\right) \Gamma\left(\frac{3}{2}\right)}=(-1)^{(p-1) / 2} p \bmod p^{2} .
$$

To achieve the congruence modulo $p^{3}$, we consider the expansion of the terminating hypergeometric series (it terminates since $(1-p) / 2$ is a negative integer)

$$
\begin{aligned}
{ }_{4} F_{3}\left[\begin{array}{c}
\frac{1}{2}(1-p), \frac{5}{4}, \frac{1}{2}(1-x), \frac{1}{2}(1+x) ;-1 \\
\frac{1}{4}, 1+\frac{1}{2} x, \quad 1-\frac{1}{2} x
\end{array}\right] \\
\quad=\sum_{k=0}^{(p-1) / 2}(4 k+1)\left(\frac{\left(\frac{1}{2}\right)_{k}}{k !}\right)^{3}(-1)^{k}+A_{2} x^{2}+\cdots \quad \text { for some } A_{2} \in \mathbb{Z}_{p} .
\end{aligned}
$$

By Lemma 2.8, if $p \mid A_{2}$, we are done. Now we follow Mortenson [2008] by using another hypergeometric evaluation identity, which is a specialization of Whipple's ${ }_{7} F_{6}$ formula (see [Bailey 1935, page 28]):

$$
\begin{aligned}
& { }_{6} F_{5}\left[\begin{array}{c}
a, 1+\frac{1}{2} a, \quad b, \quad c, \quad d, \quad e ; \quad-1 \\
\frac{1}{2} a, \quad 1+a-b, 1+a-c, 1+a-d, 1+a-e
\end{array}\right]
\end{aligned}
$$

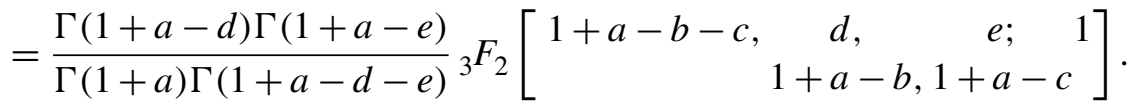


Letting $a=\frac{1}{2}, b=\frac{1-x}{2}, c=\frac{1}{2}(1+x), e=\frac{1}{2}(1-p)$ and $d=1$, we have

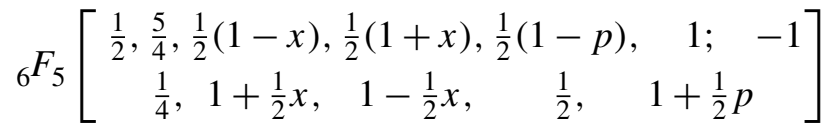

$$
\begin{aligned}
& =\frac{\Gamma\left(\frac{1}{2}\right) \Gamma\left(1+\frac{1}{2} p\right)}{\Gamma\left(\frac{3}{2}\right) \Gamma\left(\frac{1}{2} p\right)}{ }_{3} F_{2}\left[\begin{array}{ccc}
\frac{1}{2}, & 1, & \frac{1}{2}-\frac{1}{2} p ; 1 \\
1+\frac{1}{2} x, & 1-\frac{1}{2} x
\end{array}\right] .
\end{aligned}
$$

Since $\Gamma\left(\frac{1}{2}\right) \Gamma\left(1+\frac{1}{2} p\right) /\left(\left(\Gamma\left(\frac{3}{2}\right) \Gamma\left(\frac{1}{2} p\right)\right)=p\right.$, every $x$-coefficient above is in $p \mathbb{Z}_{p}$. Moreover, modulo $p$ the left side of (12) is congruent to that of (13). So when we expand the left side of (12) in terms of $x$, the coefficients are all in $p \mathbb{Z}_{p}$. In particular, $p \mid A_{2}$ and this concludes the proof of (1).

\section{Proofs of Theorems 1.1, 1.2, 1.4, and 1.5}

Whipple [1926, (7.7)] proved that

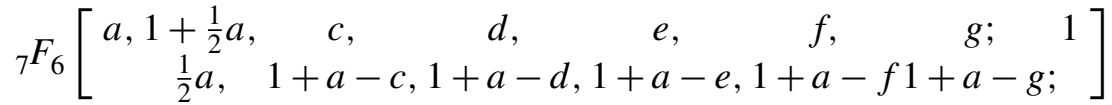

$$
\begin{aligned}
& =\frac{\Gamma(1+a-e) \Gamma(1+a-f) \Gamma(1+a-g) \Gamma(1+a-e-f-g)}{\Gamma(1+a) \Gamma(1+a-f-g) \Gamma(1+a-e-f) \Gamma(1+a-e-f)} \\
& \times{ }_{4} F_{3}\left[\begin{array}{cccc}
1+a-c-d, & e, & f, & g ; \\
& e+f+g-a, & 1+a-c, & 1+a-d
\end{array}\right],
\end{aligned}
$$

provided the ${ }_{4} F_{3}$ is a terminating series.

Proof of Theorem 1.1. Let $r$ be a positive integer and $p>3$ a prime. In (14), we let

$a=\frac{1}{2}, \quad c=\frac{1}{2}+i \frac{1}{2} p^{r}, \quad d=\frac{1}{2}-i \frac{1}{2} p^{r}, \quad e=\frac{1}{2}+\frac{1}{2} p^{r}, \quad f=\frac{1}{2}-\frac{1}{2} p^{r}, \quad g=1$, where $i=\sqrt{-1}$. Then following McCarthy and Osburn's argument, we know the left side of (14) is congruent to

$$
\sum_{k=0}^{\left(p^{r}-1\right) / 2}(4 k+1)\left(\frac{\left(\frac{1}{2}\right)_{k}}{k !}\right)^{4} \bmod p^{4 r}
$$

and the right side of (14) equals

$$
\frac{\Gamma\left(1-\frac{1}{2} p^{r}\right) \Gamma\left(1+\frac{1}{2} p^{r}\right) \Gamma\left(-\frac{1}{2}\right)}{\Gamma\left(\frac{3}{2}\right) \Gamma\left(-\frac{1}{2} p^{r}\right) \Gamma\left(\frac{1}{2} p^{r}\right)}{ }_{4} F_{3}\left[\begin{array}{ccc}
\frac{1}{2}, \frac{1}{2}+\frac{1}{2} p^{r}, \frac{1}{2}-\frac{1}{2} p^{r}, & 1 ; & 1 \\
\frac{3}{2}, & 1-i \frac{1}{2} p^{r}, 1+i \frac{1}{2} p^{r}
\end{array}\right] .
$$

Since

$$
\frac{\Gamma\left(1-\frac{1}{2} p^{r}\right) \Gamma\left(1+\frac{1}{2} p^{r}\right) \Gamma\left(-\frac{1}{2}\right)}{\Gamma\left(\frac{3}{2}\right) \Gamma\left(-\frac{1}{2} p^{r}\right) \Gamma\left(\frac{p^{r}}{2}\right)}=p^{2 r}
$$


it suffices to prove

$$
p^{r} \cdot \sum_{k=0}^{\left(p^{r}-1\right) / 2} \frac{1}{2 k+1}\left(\frac{\left(\frac{1}{2}\right)_{k}}{k !}\right)^{2} \equiv 1 \bmod p^{3} \text { for } p>3 .
$$

Recall that Lemma 2.1 says for any odd integer $n>1$,

$$
(2 n+1) \sum_{k=0}^{n} \frac{(-1)^{k}}{2 k+1}\left(\begin{array}{l}
n \\
k
\end{array}\right)\left(\begin{array}{c}
n+k \\
k
\end{array}\right)=1 .
$$

Therefore, combining this identity, congruence (8), and Lemma 2.4, we have

$$
\begin{aligned}
& p^{r} \cdot \sum_{k=0}^{\left(p^{r}-1\right) / 2} \frac{1}{2 k+1}\left(\frac{\left(\frac{1}{2}\right)_{k}}{k !}\right)^{2}=p^{r} \cdot \sum_{k=0}^{\left(p^{r}-1\right) / 2-1} \frac{1}{2 k+1}\left(\frac{\left(\frac{1}{2}\right)_{k}}{k !}\right)^{2}+\left(\frac{\left(\frac{1}{2}\right)_{\left(p^{r}-1\right) / 2}}{\left(\frac{1}{2}\left(p^{r}-1\right)\right) !}\right)^{2} \\
& \equiv p^{r} \cdot \sum_{k=0}^{\left(p^{r}-1\right) / 2-1} \frac{(-1)^{k}}{2 k+1}\left(\begin{array}{c}
\frac{1}{2}\left(p^{r}-1\right) \\
k
\end{array}\right)\left(\begin{array}{c}
\frac{1}{2}\left(p^{r}-1\right)+k \\
k
\end{array}\right) \\
& +(-1)^{\left(p^{r}-1\right) / 2}\left(\begin{array}{c}
p^{r}-1 \\
\frac{1}{2}\left(p^{r}-1\right)
\end{array}\right) \bmod p^{3} \\
& \equiv 1 \bmod p^{3} .
\end{aligned}
$$

Proof of Theorem 1.2. In (14), take

$a=\frac{1}{2}, \quad c=\frac{1}{2}+i \frac{1}{2} p, \quad d=\frac{1}{2}-i \frac{1}{2} p, \quad e=\frac{1}{2}-\frac{1}{2} p, \quad f=\frac{1}{2}+\frac{1}{2} p, \quad g=\frac{1}{2}-p^{4}$.

Then the left side of (14) is congruent to

$$
\sum_{k=0}^{(p-1) / 2}(4 k+1)\left(\frac{\left(\frac{1}{2}\right)_{k}}{k !}\right)^{6} \bmod p^{4} .
$$

Meanwhile, the right side of (14) is congruent to

$$
\begin{aligned}
& \frac{\Gamma\left(1-\frac{1}{2} p\right) \Gamma\left(1+\frac{1}{2} p\right)}{\Gamma\left(\frac{1}{2}\right) \Gamma\left(\frac{3}{2}\right)} \frac{\Gamma\left(1+p^{4}\right) \Gamma\left(p^{4}\right)}{\Gamma\left(\frac{1}{2}+\frac{1}{2} p+p^{4}\right) \Gamma\left(\frac{1}{2}-\frac{1}{2} p+p^{4}\right)} \\
& \times \sum_{k=0}^{(p-1) / 2} \frac{\left(\frac{1}{2}\right)_{k}^{2}\left(\frac{1}{2}+\frac{1}{2} p\right)_{k}\left(\frac{1}{2}-\frac{1}{2} p\right)_{k}}{k !^{2}\left(1-i \frac{1}{2} p\right)_{k}\left(1+i \frac{1}{2} p\right)_{k}} \bmod p^{4},
\end{aligned}
$$

where

$$
\frac{\Gamma\left(1-\frac{1}{2} p\right) \Gamma\left(1+\frac{1}{2} p\right)}{\Gamma\left(\frac{1}{2}\right) \Gamma\left(\frac{3}{2}\right)}=(-1)^{(p-1) / 2} p
$$


and

$$
\begin{aligned}
& \frac{\Gamma\left(1+p^{4}\right) \Gamma\left(p^{4}\right)}{\Gamma\left(\frac{1}{2}+\frac{1}{2} p+p^{4}\right) \Gamma\left(\frac{1}{2}-\frac{1}{2} p+p^{4}\right)}=\frac{\left(p^{4}-\frac{1}{2}(p-1)\right)_{(p-1) / 2}}{\left(1+p^{4}\right)_{(p-1) / 2}} \\
& \quad \equiv \frac{\left(-\frac{1}{2}(p-1)\right)\left(-\frac{1}{2}(p-1)+1\right) \cdots(-1)}{1 \cdot 2 \cdots\left(\frac{1}{2}(p-1)\right)} \bmod p=(-1)^{(p-1) / 2} .
\end{aligned}
$$

Therefore, Theorem 1.2 follows from the result of Kilbourn (see (3)) and the next lemma.

Lemma 4.1. Let $p>3$ be a prime, then

$$
\sum_{k=0}^{(p-1) / 2} \frac{\left(\frac{1}{2}\right)_{k}^{2}\left(\frac{1}{2}+\frac{1}{2} p\right)_{k}\left(\frac{1}{2}-\frac{1}{2} p\right)_{k}}{k !^{2}\left(1-i \frac{1}{2} p\right)_{k}\left(1+i \frac{1}{2} p\right)_{k}} \equiv \sum_{k=0}^{(p-1) / 2}\left(\frac{\left(\frac{1}{2}\right)_{k}}{k !}\right)^{4} \bmod p^{3}
$$

Proof. Expand

$$
\sum_{k=0}^{(p-1) / 2} \frac{\left(\frac{1}{2}\right)_{k}^{2}\left(\frac{1}{2}+\frac{1}{2} x\right)_{k}\left(\frac{1}{2}-\frac{1}{2} x\right)_{k}}{k !^{2}\left(1-i \frac{1}{2} x\right)_{k}\left(1+i \frac{1}{2} x\right)_{k}}=\sum_{k=0}^{(p-1) / 2}\left(\frac{\left(\frac{1}{2}\right)_{k}}{k !}\right)^{4}\left(1+b_{2, k} x^{2}+b_{4, k} x^{4}+\cdots\right) .
$$

Using (8) and (9), we have

$$
b_{2, k}=-\sum_{j=1}^{k} \frac{1}{(2 j-1)^{2}}-\frac{1}{4} \sum_{j=1}^{k} \frac{1}{j^{2}}=-\sum_{j=1}^{2 k} \frac{1}{j^{2}} .
$$

The claim is verified by using Lemma 2.8 and taking $s=2$ in Lemma 2.7.

Proof of Theorem 1.4. We start with the following combinatorial identity.

Lemma 4.2. $\quad \sum_{k=0}^{(p-1) / 2}(6 k+1) \frac{\left(\frac{1}{2}\right)_{k}\left(\frac{1}{2}-\frac{1}{2} p\right)_{k}\left(\frac{1}{2}+\frac{1}{2} p\right)_{k}}{(1)_{k}\left(1+\frac{1}{4} p\right)_{k}\left(1-\frac{1}{4} p\right)_{k}} \frac{1}{4^{k}}=(-1)^{(p-1) / 2} p$.

Proof. Recall that [Gessel 1995, (31.1)] says

$$
\begin{array}{r}
{ }_{5} F_{4}\left[\begin{array}{rrr}
\frac{1}{2}+a-c, \quad-n, & n+1, & 2-2 c+n, \frac{5}{3}-\frac{2}{3} c+\frac{1}{3} n ; \frac{1}{4} \\
2-c+n, \frac{2}{3}-\frac{2}{3} c+\frac{1}{3} n, n-2 a+2, & \frac{3}{2}-c
\end{array}\right] \\
=\frac{(2-c)_{n}(2-2 a)_{n}}{(3-2 c)_{n}\left(\frac{3}{2}-a\right)_{n}} .
\end{array}
$$
have

Letting $a=\frac{1}{2}+\frac{1}{4} p, c=\frac{1}{2}+\frac{1}{4} p$, and $n=\frac{1}{2}(p-1)$ and using Lemma 2.2, we

$$
\begin{aligned}
{ }_{5} F_{4}\left[\begin{array}{c}
\frac{1}{2}, \frac{1}{2}, \frac{7}{6}, \frac{1}{2}-\frac{1}{2} p, \frac{1}{2}+\frac{1}{2} p ; \frac{1}{4} \\
\frac{1}{2}, \frac{1}{6}, 1-\frac{1}{4} p, 1+\frac{1}{4} p
\end{array}\right] & =\frac{\left(\frac{3}{2}-\frac{1}{4} p\right)_{(p-1) / 2}\left(1-\frac{1}{2} p\right)_{(p-1) / 2}}{\left(2-\frac{1}{2} p\right)_{(p-1) / 2}\left(1-\frac{1}{4} p\right)_{(p-1) / 2}} \\
& =(-1)^{(p-1) / 2} p .
\end{aligned}
$$




\section{Lemma 4.3. The function}

$$
\left(\sum_{k=0}^{(p-1) / 2}(6 k+1) \frac{\left(\frac{1}{2}\right)_{k}\left(\frac{1}{2}-\frac{1}{2} x\right)_{k}\left(\frac{1}{2}+\frac{1}{2} x\right)_{k}}{(1)_{k}\left(1+\frac{1}{4} x\right)_{k}\left(1-\frac{1}{4} x\right)_{k}} \frac{1}{4^{k}}\right) /\left(\sum_{k=0}^{(p-1) / 2} \frac{6 k+1}{4^{k}}\left(\frac{\left(\frac{1}{2}\right)_{k}}{k !}\right)^{3}\right)
$$

is a formal power series in $x^{2}$ with coefficients in $\mathbb{Z}_{p}$. Its $x^{2}$ coefficient is zero modulo $p$.

Proof. We use the strange valuation of Gosper:

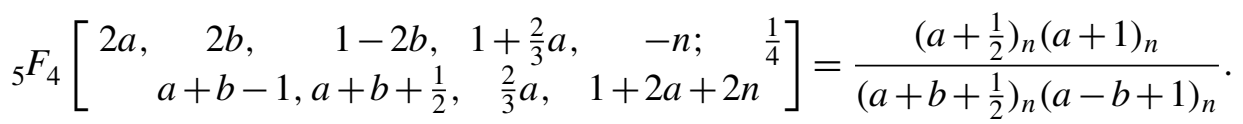

See [Gessel and Stanton 1982, (1.2)]. Let $a=\frac{1}{4}, b=\frac{1}{4}-\frac{1}{4} x$ and $n=\frac{1}{2}(p-1)$. Then the left side of the above equals

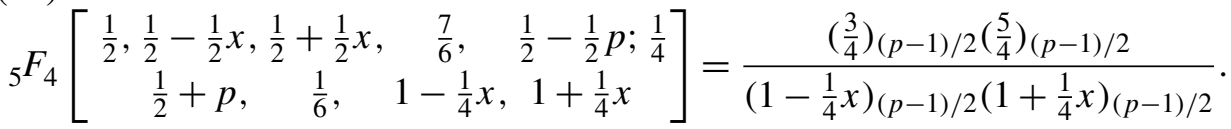

We remark that

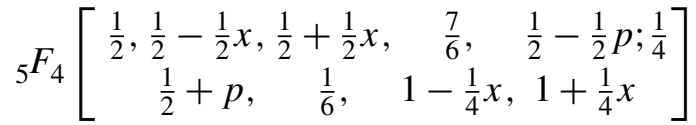

$$
\begin{aligned}
& \equiv \sum_{k=0}^{(p-1) / 2} \frac{6 k+1}{4^{k}} \frac{\left(\frac{1}{2}\right)_{k}\left(\frac{1}{2}-\frac{1}{2} x\right)_{k}\left(\frac{1}{2}+\frac{1}{2} x\right)_{k}}{(1)_{k}\left(1+\frac{1}{4} x\right)_{k}\left(1-\frac{1}{4} x\right)_{k}} \quad \bmod p .
\end{aligned}
$$

When $x=0$, the right hand side of (15) equals $\left(\frac{3}{4}\right)_{(p-1) / 2}\left(\frac{5}{4}\right)_{(p-1) / 2} /(1)_{(p-1) / 2}^{2}$, which is in $p \mathbb{Z}_{p}$. In fact, if $p \equiv 1 \bmod 4$ then $\frac{5}{4}+\frac{1}{4}(p-1)-1=\frac{1}{4} p$, and if $p \equiv$ $3 \bmod 4$, then $\frac{3}{4}+\frac{1}{4}(p-3)=\frac{1}{4} p$, while $(1)_{(p-1) / 2}$ is a $p$-adic unit. It is not difficult to see that $p$ divides $((3) / 4)_{(p-1) / 2}\left(\frac{5}{4}\right)_{(p-1) / 2} /(1)_{(p-1) / 2}^{2}$ exactly. Consequently, if we expand

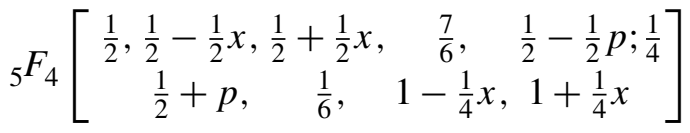

in terms of formal power series of $x$ (in fact, $x^{2}$ ), each coefficient is in $p \mathbb{Z}_{p}$. Thus the coefficients of the right side of (16), including the coefficient of $x^{2}$, are all divisible by $p$. By Lemmas 2.8 and 4.2 ,

$$
\sum_{k=0}^{(p-1) / 2} \frac{6 k+1}{4^{k}}\left(\frac{\left(\frac{1}{2}\right)_{k}}{k !}\right)^{3} \equiv(-1)^{(p-1) / 2} p \bmod p^{3} .
$$


Namely,

$$
\sum_{k=0}^{(p-1) / 2} \frac{6 k+1}{4^{k}}\left(\frac{\left(\frac{1}{2}\right)_{k}}{k !}\right)^{3}=(-1)^{(p-1) / 2} p+a p^{3} \quad \text { for some } a \in \mathbb{Z}_{p} .
$$

The statement of Theorem 1.4 is equivalent to $a \in p \mathbb{Z}_{p}$.

The quotient

$$
\left(\sum_{k=0}^{(p-1) / 2} \frac{6 k+1}{4^{k}} \frac{\left(\frac{1}{2}\right)_{k}\left(\frac{1}{2}-\frac{1}{2} x\right)_{k}\left(\frac{1}{2}+\frac{1}{2} x\right)_{k}}{(1)_{k}\left(1+\frac{1}{4} x\right)_{k}\left(1-\frac{1}{4} x\right)_{k}}\right) /\left(\sum_{k=0}^{(p-1) / 2} \frac{6 k+1}{4^{k}} \frac{\left(\frac{1}{2}\right)_{k}}{(1)_{k}}\right)
$$

is a formal power series in $x^{2}$ with $p$-integral coefficients, since the denominators are divisible by $p$ exactly. The same conclusion applies to

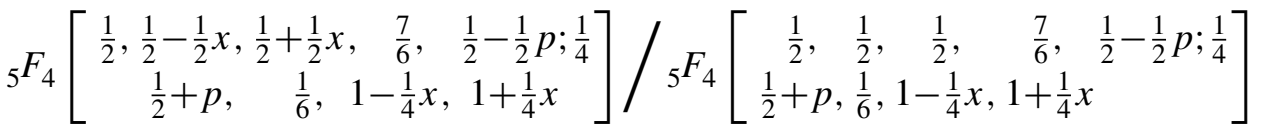

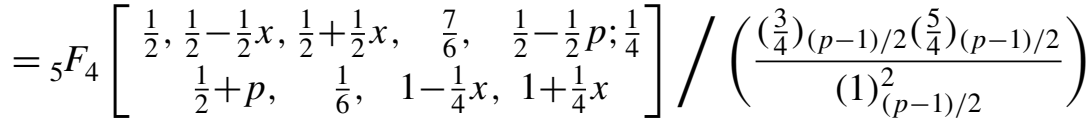

$$
\begin{aligned}
& =\frac{(1)_{(p-1) / 2}^{2}}{\left(1-\frac{1}{4} x\right)_{(p-1) / 2}\left(1+\frac{1}{4} x\right)_{(p-1) / 2}} \text {. }
\end{aligned}
$$

On the other hand, by (9), the $x^{2}$ coefficient of

$$
\frac{(1)_{(p-1) / 2}^{2}}{\left(1-\frac{1}{4} x\right)_{(p-1) / 2}\left(1+\frac{1}{4} x\right)_{(p-1) / 2}}
$$

is a scalar multiple of $H_{(p-1) / 2}^{(2)}$, which is in $p \mathbb{Z}_{p}$ by Lemma 2.5; so is the $x^{2}$ coefficient of (17).

By Lemma 2.8 and the analysis above,

$$
\frac{(-1)^{(p-1) / 2} p}{(-1)^{(p-1) / 2} p+a p^{3}}=\frac{(-1)^{(p-1) / 2}}{(-1)^{(p-1) / 2}+a p^{2}} \equiv 1 \bmod p^{3}
$$

hence $a \in p \mathbb{Z}_{p}$, which concludes the proof of Theorem 1.4.

\section{Lemma 4.4.}

$$
\sum_{k=0}^{(p-1) / 2}(6 k+1) \frac{\left(\frac{1}{2}\right)_{k}\left(\frac{1}{2}-\frac{1}{2} p\right)_{k}\left(\frac{1}{2}+\frac{1}{2} p\right)_{k}}{(1)_{k}\left(1+\frac{1}{4} p\right)_{k}\left(1-\frac{1}{4} p\right)_{k}} \frac{(-1)^{k}}{8^{k}}=(-1)^{\left(p^{2}-1\right) / 8+(p-1) / 2} p .
$$


Proof. This time, we use [Gessel 1995, last identity of page 544]

$$
{ }_{4} F_{3}\left[\begin{array}{c}
2 a+n+1, \quad n+1, \quad \frac{2}{3} a+\frac{1}{3} n+\frac{4}{3},-n ;-\frac{1}{8} \\
a+\frac{3}{2}+n, \frac{2}{3} a+\frac{1}{3} n+\frac{1}{3}, 1+a
\end{array}\right]=\frac{\left(a+\frac{3}{2}\right)_{n}}{(2 a+2)_{n}} 2^{n} .
$$

Letting $a=-\frac{1}{4} p$ and $n=\frac{1}{2}(p-1)$ and using Lemma 2.3, we have

$$
\begin{aligned}
{ }_{4} F_{3}\left[\begin{array}{c}
\frac{1}{2}, \frac{7}{6}, \frac{1}{2}+\frac{1}{2} p, \frac{1}{2}-\frac{1}{2} p ;-\frac{1}{8} \\
\frac{1}{6}, 1-\frac{1}{4} p, 1+\frac{1}{4} p
\end{array}\right] & =\frac{\left(\frac{3}{2}-\frac{1}{4} p\right)_{(p-1) / 2}}{\left(2-\frac{1}{2} p\right)_{(p-1) / 2}} 2^{(p-1) / 2} \\
& =(-1)^{\left(p^{2}-1\right) / 8+(p-1) / 2} p .
\end{aligned}
$$

Proof of Theorem 1.5. Equation (5) is a consequence of Lemma 4.4.

Remark 1. Van Hamme's conjecture that

$$
\sum_{k=0}^{(p-1) / 2}(6 k+1)\left(\frac{\left(\frac{1}{2}\right)_{k}}{k !}\right)^{3} \frac{(-1)^{k}}{8^{k}} \equiv(-1)^{\left(p^{2}-1\right) / 8+(p-1) / 2} p \bmod p^{3}
$$

holds if

$$
\sum_{k=0}^{(p-1) / 2}(6 k+1)\left(\frac{\left(\frac{1}{2}\right)_{k}}{k !}\right)^{3}\left(\sum_{j=1}^{k} \frac{1}{(2 j-1)^{2}}-\frac{1}{16} \sum_{j=1}^{k} \frac{1}{j^{2}}\right) \frac{(-1)^{k}}{8^{k}} \equiv 0 \bmod p .
$$

The proof of the latter is left to the interested reader.

Remark 2. In [2009], Zudilin proved the congruence (2) modulo $p^{2}$ and the congruence (5) modulo $p$.

\section{Acknowledgments}

I would like to thank Heng Huat Chan and Wadim Zudilin for their encouragement, enlightening discussions and valuable comments. In particular, Zudilin pointed me to a few useful ideas and the reference [Gessel and Stanton 1982]. I further thank the anonymous referees for their detailed comments, including pointing out the reference [Cai 2002], on an earlier version of this article.

\section{References}

[Ahlgren and Ono 2000] S. Ahlgren and K. Ono, "A Gaussian hypergeometric series evaluation and Apéry number congruences”, J. Reine Angew. Math. 518 (2000), 187-212. MR 2001c:11057 Zbl 0940.33002

[Bailey 1935] W. N. Bailey, Generalized hypergeometric series, Cambridge Tracts in Mathematics and Mathematical Physics 32, Cambridge University Press, London, 1935. MR 32 \#2625 Zbl 0011.02303

[Cai 2002] T. Cai, "A congruence involving the quotients of Euler and its applications, I", Acta Arith. 103:4 (2002), 313-320. MR 2003d:11007 Zbl 1008.11001 
[Chan et al. 2010] H. H. Chan, L. Long, and W. Zudilin, "A supercongruence motivated by the Legendre family of elliptic curves", Mat. Zametki 88:4 (2010), 621-625. In Russian; translated in Mathematical Notes 88:4 (2010), 599-602.

[Gessel 1995] I. M. Gessel, "Finding identities with the WZ method", J. Symbolic Comput. 20:5-6 (1995), 537-566. MR 97j:05010 Zbl 0908.33004

[Gessel and Stanton 1982] I. Gessel and D. Stanton, "Strange evaluations of hypergeometric series", SIAM J. Math. Anal. 13:2 (1982), 295-308. MR 83c:33002 Zbl 0486.33003

[van Hamme 1997] L. van Hamme, "Some conjectures concerning partial sums of generalized hypergeometric series", pp. 223-236 in p-adic functional analysis (Nijmegen, 1996), edited by W. H. Schikhof et al., Lecture Notes in Pure and Appl. Math. 192, Dekker, New York, 1997. MR 98k:33011 Zbl 0895.11051

[Kilbourn 2006] T. Kilbourn, "An extension of the Apéry number supercongruence", Acta Arith. 123:4 (2006), 335-348. MR 2007e:11049 Zbl 1170.11008

[McCarthy 2009] D. McCarthy, "Supercongruence conjectures of Rodriguez-Villegas", preprint, 2009. arXiv 0907.5089

[McCarthy and Osburn 2008] D. McCarthy and R. Osburn, "A $p$-adic analogue of a formula of Ramanujan”, Arch. Math. (Basel) 91:6 (2008), 492-504. MR 2009k:11191 Zbl 1175.33004

[Morley 1895] F. Morley, "Note on the congruence $2^{4 n} \equiv(-1)^{n}(2 n) ! /(n !)^{2}$, where $2 n+1$ is prime", Annals of Math. 9 (1895), 168-170.

[Mortenson 2008] E. Mortenson, "A p-adic supercongruence conjecture of van Hamme", Proc. Amer. Math. Soc. 136:12 (2008), 4321-4328. MR 2010g:11203 Zbl 1171.11061

[Rodriguez-Villegas 2003] F. Rodriguez-Villegas, "Hypergeometric families of Calabi-Yau manifolds", pp. 223-231 in Calabi-Yau varieties and mirror symmetry (Toronto, ON, 2001), edited by N. Yui and J. D. Lewis, Fields Inst. Commun. 38, Amer. Math. Soc., Providence, RI, 2003. MR 2005b:11086 Zbl 1062.11038

[Whipple 1926] F. J. W. Whipple, "On well-posed series, generalised hypergeometric series having parameters in pairs, each pair with the same sum", Proc. London Math. Soc. 24:1 (1926), 247-263.

[Zudilin 2009] W. Zudilin, "Ramanujan-type supercongruences", J. Number Theory 129:8 (2009), 1848-1857. MR 2522708 Zbl 05569026

Received December 1, 2009. Revised September 27, 2010.

LING LONG

DEPARTMENT OF MATHEMATICS

IOWA STATE UNIVERSITY

396 CARVER HALL

AMES, IA 50011

UNITED STATES

linglong@iastate.edu

http://www.math.iastate.edu/linglong 


\title{
PACIFIC JOURNAL OF MATHEMATICS
}

\author{
http://www.pjmath.org \\ Founded in 1951 by
}

E. F. Beckenbach (1906-1982) and F. Wolf (1904-1989)

\section{EDITORS}

V. S. Varadarajan (Managing Editor)

Department of Mathematics

University of California

Los Angeles, CA 90095-1555

pacific@math.ucla.edu

Vyjayanthi Chari

Department of Mathematics

University of California

Riverside, CA 92521-0135

chari@math.ucr.edu

\section{Robert Finn}

Department of Mathematics Stanford University

Stanford, CA 94305-2125

finn@math.stanford.edu

Kefeng Liu

Department of Mathematics

University of California

Los Angeles, CA 90095-1555

liu@math.ucla.edu
Darren Long

Department of Mathematics

University of California

Santa Barbara, CA 93106-3080

long@math.ucsb.edu

Jiang-Hua Lu

Department of Mathematics

The University of Hong Kong

Pokfulam Rd., Hong Kong jhlu@maths.hku.hk

Alexander Merkurjev

Department of Mathematics University of California

Los Angeles, CA 90095-1555 merkurev@math.ucla.edu
Sorin Popa

Department of Mathematics

University of California

Los Angeles, CA 90095-1555

popa@math.ucla.edu

Jie Qing

Department of Mathematics

University of California

Santa Cruz, CA 95064

qing@ cats.ucsc.edu

Jonathan Rogawski

Department of Mathematics

University of California

Los Angeles, CA 90095-1555

jonr@math.ucla.edu

\section{PRODUCTION}

pacific@math.berkeley.edu

Silvio Levy, Scientific Editor Matthew Cargo, Senior Production Editor

ACADEMIA SINICA, TAIPEI

CALIFORNIA INST. OF TECHNOLOGY

INST. DE MATEMÁTICA PURA E APLICADA

KEIO UNIVERSITY

MATH. SCIENCES RESEARCH INSTITUTE

NEW MEXICO STATE UNIV.

OREGON STATE UNIV.

\section{SUPPORTING INSTITUTIONS}

STANFORD UNIVERSITY
UNIV. OF BRITISH COLUMBIA
UNIV. OF CALIFORNIA, BERKELEY
UNIV. OF CALIFORNIA, DAVIS
UNIV. OF CALIFORNIA, LOS ANGELES
UNIV. OF CALIFORNIA, RIVERSIDE
UNIV. OF CALIFORNIA, SAN DIEGO
UNIV. OF CALIF., SANTA BARBARA

UNIV. OF CALIF., SANTA CRUZ

UNIV. OF MONTANA

UNIV. OF OREGON

UNIV. OF SOUTHERN CALIFORNIA

UNIV. OF UTAH

UNIV. OF WASHINGTON

WASHINGTON STATE UNIVERSITY

These supporting institutions contribute to the cost of publication of this Journal, but they are not owners or publishers and have no responsibility for its contents or policies.

See inside back cover or www.pjmath.org for submission instructions.

The subscription price for 2011 is US \$420/year for the electronic version, and \$485/year for print and electronic.

Subscriptions, requests for back issues from the last three years and changes of subscribers address should be sent to Pacific Journal of Mathematics, P.O. Box 4163, Berkeley, CA 94704-0163, U.S.A. Prior back issues are obtainable from Periodicals Service Company, 11 Main Street, Germantown, NY 12526-5635. The Pacific Journal of Mathematics is indexed by Mathematical Reviews, Zentralblatt MATH, PASCAL CNRS Index, Referativnyi Zhurnal, Current Mathematical Publications and the Science Citation Index.

The Pacific Journal of Mathematics (ISSN 0030-8730) at the University of California, c/o Department of Mathematics, 969 Evans Hall, Berkeley, CA 94720-3840, is published monthly except July and August. Periodical rate postage paid at Berkeley, CA 94704, and additional mailing offices. POSTMASTER: send address changes to Pacific Journal of Mathematics, P.O. Box 4163, Berkeley, CA 94704-0163.

PJM peer review and production are managed by EditFLOW ${ }^{\mathrm{TM}}$ from Mathematical Sciences Publishers.

PUBLISHED BY PACIFIC JOURNAL OF MATHEMATICS

at the University of California, Berkeley 94720-3840

A NON-PROFIT CORPORATION

Typeset in IATEX

Copyright $(2011$ by Pacific Journal of Mathematics 


\section{PACIFIC JOURNAL OF MATHEMATICS}

Volume $249 \quad$ No. $2 \quad$ February 2011

A gluing construction for prescribed mean curvature

257

ADRIAN BUTSCHER

Large eigenvalues and concentration

271

BRUNO COLBOIS and ALESSANDRO SAVO

Sur les conditions d'existence des faisceaux semi-stables sur les courbes multiples primitives

JEAN-MARC DRÉZET

A quantitative estimate for quasiintegral points in orbits

LIANG-CHUNG HSIA and JosePh H. Silverman

Möbius isoparametric hypersurfaces with three distinct principal curvatures, II

ZEJUN HU and SHUJIE ZHAI

Discrete Morse theory and Hopf bundles

371

DMITRY N. KOZLOV

Regularity of canonical and deficiency modules for monomial ideals

ManOJ KUMmini and SATOSHI MURAI

$\mathrm{SL}_{2}(\mathbb{C})$-character variety of a hyperbolic link and regulator

WEIPING LI and QINGXUE WANG

Hypergeometric evaluation identities and supercongruences

LING LONG

Necessary and sufficient conditions for unit graphs to be Hamiltonian

H. R. Maimani, M. R. Pournaki and S. Yassemi

Instability of the geodesic flow for the energy functional

DOMENico PERrone

String structures and canonical 3-forms

CORBETT REDDEN

Dual pairs and contragredients of irreducible representations

BINYONG SUN

On the number of pairs of positive integers $x_{1}, x_{2} \leq H$ such that $x_{1} x_{2}$ is a $k$-th power

DOYCHIN I. TOLEV

Correction to the article A Floer homology for exact contact embeddings 